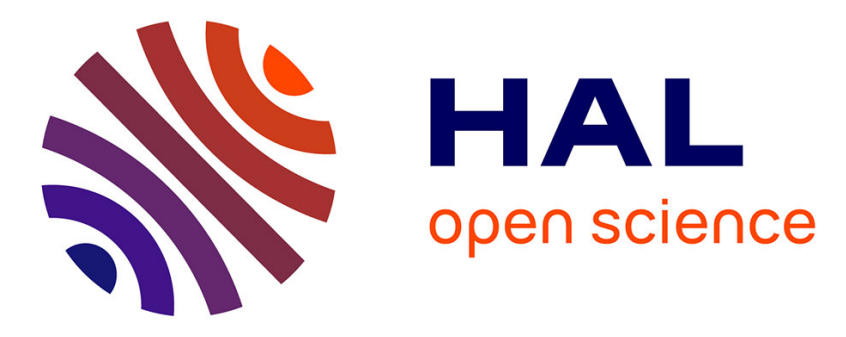

\title{
Feature Extraction on Local Jet Space for Texture Classification
}

Marcos William da Silva Oliveira, Núbia Rosa da Silva, Antoine Manzanera, Odemir Martinez Bruno

\section{- To cite this version:}

Marcos William da Silva Oliveira, Núbia Rosa da Silva, Antoine Manzanera, Odemir Martinez Bruno. Feature Extraction on Local Jet Space for Texture Classification. Physica A: Statistical Mechanics and its Applications, 2015, 10.1016/j.physa.2015.06.046 . hal-01245094

\section{HAL Id: hal-01245094 \\ https://hal.science/hal-01245094}

Submitted on 16 Dec 2015

HAL is a multi-disciplinary open access archive for the deposit and dissemination of scientific research documents, whether they are published or not. The documents may come from teaching and research institutions in France or abroad, or from public or private research centers.
L'archive ouverte pluridisciplinaire HAL, est destinée au dépôt et à la diffusion de documents scientifiques de niveau recherche, publiés ou non, émanant des établissements d'enseignement et de recherche français ou étrangers, des laboratoires publics ou privés. 


\title{
Feature Extraction on Local Jet Space for Texture Classification
}

\author{
Marcos William da Silva Oliveira ${ }^{\mathrm{a}, \mathrm{b}}$, Núbia Rosa da Silva ${ }^{\mathrm{a}, \mathrm{b}}$, Antoine \\ Manzanera $^{c}$, Odemir Martinez Brunob,a \\ ${ }^{a}$ Institute of Mathematics and Computer Science, University of São Paulo, USP, \\ Avenida Trabalhador são-carlense, 400, 13566-590 São Carlos, São Paulo, Brazil \\ ${ }^{b}$ Scientific Computing Group, São Carlos Institute of Physics, University of São Paulo, \\ USP, cx 369 13560-970 São Carlos, São Paulo, Brazil - www.scg.ifsc.usp.br \\ ${ }^{c}$ ENSTA-ParisTech, U2IS-Robotics \& Vision, Université de Paris-Saclay, 828, \\ Boulevard des Maréchaux, 91762 Palaiseau CEDEX, France
}

\begin{abstract}
The proposal of this study is to analyze the texture pattern recognition over the local jet space looking forward to improve the texture characterization. Local jets decompose the image based on partial derivatives allowing the texture feature extraction be exploited in different levels of geometrical structures. Each local jet component evidences a different local pattern, such as, flat regions, directional variations and concavity or convexity. Subsequently, a texture descriptor is used to extract features from 0th, 1st and 2nd-derivative components. Four well-known databases (Brodatz, Vistex, Usptex and Outex) and four texture descriptors (Fourier descriptors, Gabor filters, Local Binary Pattern and Local Binary Pattern Variance) were used to validate the idea, showing in most cases an increase of the success rates.
\end{abstract}

Keywords: Local jet space, pattern recognition, texture, image analysis

\section{Introduction}

Local jets make a decomposition of the image, that is an intensity function, based on partial derivatives $[1,2,3]$. This approach can be seen as a model of receptive fields of human cortex [4]. Receptive fields are able to discriminate geometric characteristics of the visual scene like lines, edges, orientations, or curvatures. In [3], a local jet space framework was developed 
to process and represent images extracting relevant information over the decomposition of the image by a set of functions, demonstrating its efficiency in optical flow estimation, Non-Local means filtering, background subtraction and image and object characterization. Moreover, this framework can be used in a wide range of applications, such as real time video processing, detecting, predicting and filtering.

In the context of image representation, the behavior of local jet can be used to highlight the geometric characteristics of texture patterns. Furthermore, the local jet decomposition creates a similarity space of image that can be explored to classify texture images. Texture features have been played a significant role in pattern recognition and image analysis, including applications using real and synthetic images in several fields, for example plant analysis and identification $[5,6,7,8]$, medical image analysis $[9,10,11]$ and many others. Aiming to increase the potential of traditional texture descriptors, our proposal is to enhance the texture feature extraction process using the local jet decomposition as initial transformation. The texture image is represented by a set of features composed by the result of the application of a texture descriptor on the local jet components.

The performance of the proposal is assessed over four databases: Brodatz [12], Usptex [13], Vistex [14], and Outex [15]; using four texture feature descriptors: Fourier, Gabor, Local Binary Pattern and Local Binary Pattern Variance. A principal component analysis (PCA) [16] was performed to reduce the dimension of features and linear discriminant analysis (LDA) is used to perform the classification. In most experiments, the proposed approach obtained higher success rate compared to the feature descriptors applied without the local jet decomposition.

The remainder of this paper is organized as follows. Section 2 presents the theory of local jet. Section 3 describes how is the process of feature extraction in the local jet space as well as each of the texture extractors used in the experiments. Section 4 outlines the experiments to validate the proposal, detailing the databases and procedures for classification. The results and discussion of the classification experiments are reported in Section 5, and, finally, conclusions are presented in Section 6 .

\section{Local Jet}

To extract the basic local representation of images, we have used the multiscale local jet, which can be used as a similarity space [2], based on the 
approximation provided by the Taylor expansion. For a function $f: \mathbb{R}^{2} \rightarrow \mathbb{R}$, at order $r$, the Taylor expansion is

$$
f\left(x+c_{1}, y+c_{2}\right)=\sum_{k=0}^{r} \sum_{i=0}^{k}\left(\begin{array}{l}
k \\
i
\end{array}\right) c_{1}^{k-i} c_{2}^{i} \frac{\partial^{k} f}{\partial x^{i} \partial y^{k-i}}(x, y)+\mathcal{O}\left(\|\mathbf{c}\|^{r}\right),
$$

where $\mathbf{c}=\left(c_{1}, c_{2}\right)$ is the residual. The notation of partial derivatives can be changed to $f_{i j}=\frac{\partial^{k} f}{\partial x^{i} \partial y^{k-i}}$. In image processing context, $f: \Omega \in \mathbb{R}^{2} \rightarrow \mathbb{R}$ is the original image and the derivatives are estimated at a certain scale [1], by convolving the image with Gaussian derivatives, as

$$
f_{i j}^{\sigma}=f \star \frac{\partial^{i+j} G_{\sigma}}{\partial x^{i} \partial y^{j}}
$$

where $G_{\sigma}$ is the bi-dimensional Gaussian filter with standard deviation $\sigma$, which represents the estimation scale. Hence, the local jet space is the set $\left\{f_{i j}^{\sigma} \mid i+j \leqslant r, \sigma \in S\right\}$. In this work, the scale parameter is set to 1 (i.e. only one scale is used), and the order of derivation is set to 2 . Thus, the local jet space of image $f: \Omega \in \mathbb{R}^{2} \rightarrow \mathbb{R}$ is the collection $\left\{f_{00}, f_{10}, f_{01}, f_{20}, f_{02}, f_{11}\right\}$. Figure 1 shows this decomposition applied to a texture image.

Each image in local jet space highlights different geometric properties of scene image. Figure 2 shows the isolines of a texture image and the isolines of some local jet components. We can see that the peaks in original image is blurred in $f_{00}$ component in which flat regions can be better analyzed. On $f_{10}$ resulting image, the intensity of directional variation is evidenced and the intensities in second order components represent the measure of concavity or convexity of each pixel neighbor on the image surface, emphasizing the different local variations.

\section{Texture Feature Extraction on Local Jet Space}

The primary goal of the proposed approach was to emphasize the intrinsic content in texture images that can not be extracted directly from the original image. The local jet decomposition enhances geometric properties in texture images to improve the action of feature extractors.

The flow in Figure 3 summarizes the idea where local jet decomposition until the second order on Equation(2) is applied in all images of the database, i.e., six local jet components are calculated for each image. A texture feature 


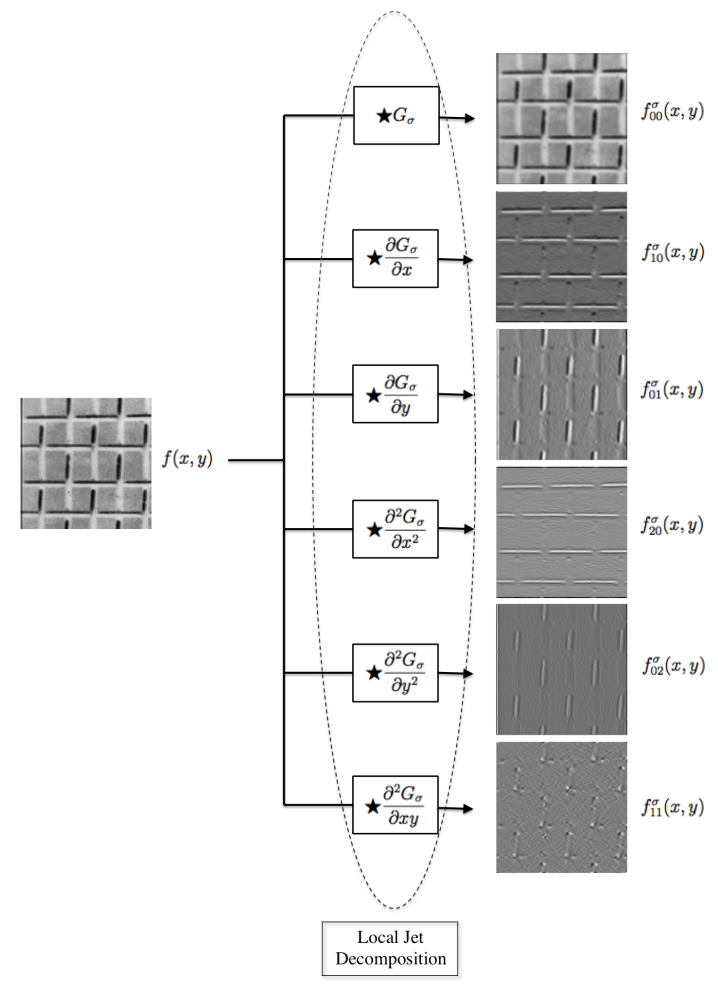

Figure 1: Local jet decomposition of one Brodatz texture image based on Taylor expansion.

extractor is used on these local jet components characterizing each image in six $n$-dimensional feature vectors. The dimension $n$ of each feature vector is dependent on the feature extractor. The PCA transformation is applied to the resulting set of feature vectors of each texture descriptor. This procedure aims to find the directions of the feature space along which the variability of feature vectors is maximal, allowing to reduce the dimension of the feature space.

Different feature extractors are used in this approach based on their different analysis of geometric properties. The application of this methodology is not limited to these feature extractors. They were chosen due to the wellknown applications of these descriptors in the literature. In the remainder, each descriptor is presented. 


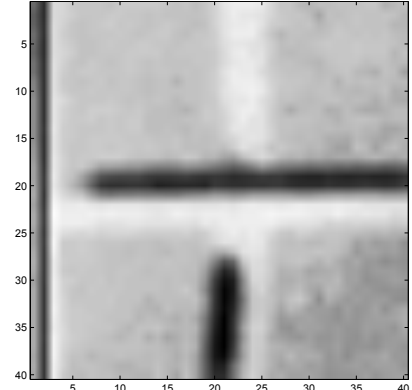

$\left(a^{2}\right)$

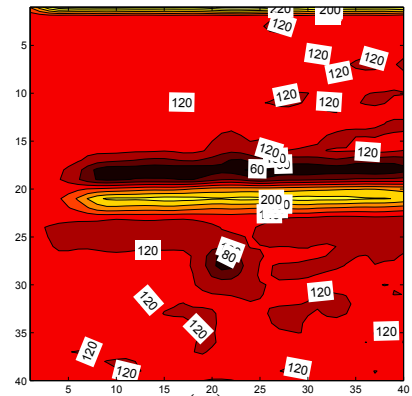

$(\mathrm{d})$

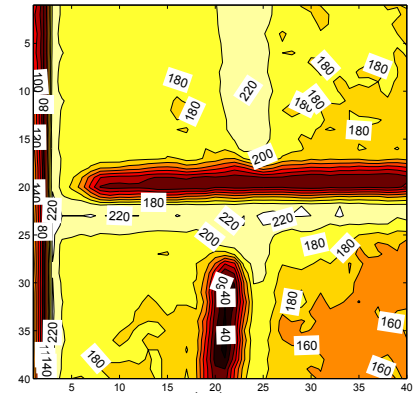

$\left(b^{20}\right)$

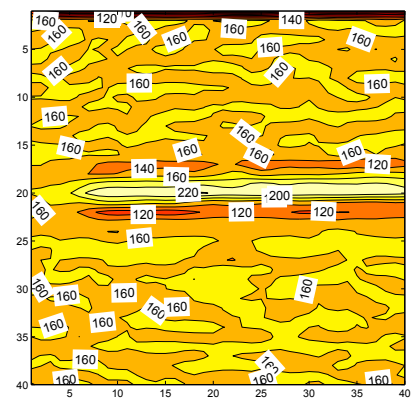

$(\mathrm{e})$

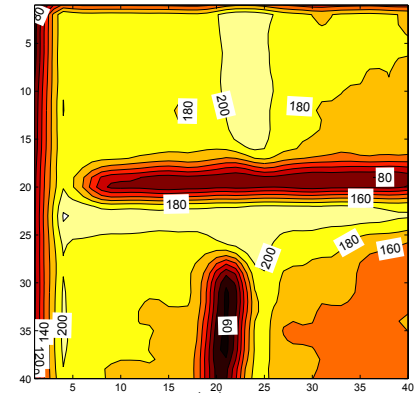

$(\mathrm{c})$

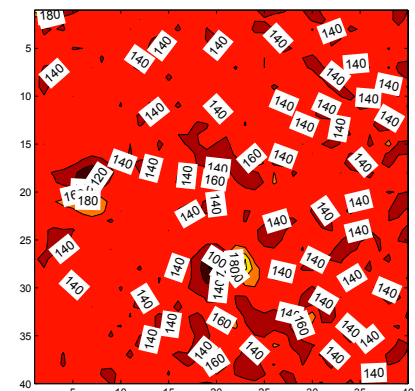

$(\mathrm{f})$

Figure 2: Isolines of local jet decomposition. (a) A cropping of one image form Brodatz database and its original image isolines (b). Isolines of (a): (c) $f_{00},(\mathrm{~d}) f_{10}$, (e) $f_{20}$ and (f) $f_{11}$.

\subsection{Fourier descriptors}

The Fourier descriptors was proposed originally by Cosgiff in 1960 [17] to caracterize a periodic signal. For texture classification, Azencott et al. [18] extract texture features based on windowed Fourier filters.

The basic idea behind Fourier descriptors is that texture features are extracted on the frequency domain. Then, this process is performed by the application of 2D Fourier transform on the texture image $f(x, y)$ multiplied by $(-1)^{x+y}$ to shift the origin of $F(u, v)$ spectrum to center of the area occupied by the frequency domain [19] as

$$
\mathfrak{F}\left[f(x, y)(-1)^{x+y}\right]=F(u-M / 2, v-N / 2),
$$

where $M \times N$ is the size of image. This procedure allows the use of concentric circles on $(0,0)$ to obtain the features.

Based on $[19,20]$, the feature vector is composed by two different characteristics extracted from spectrum: $E a$ is the energy extracted from sectors 


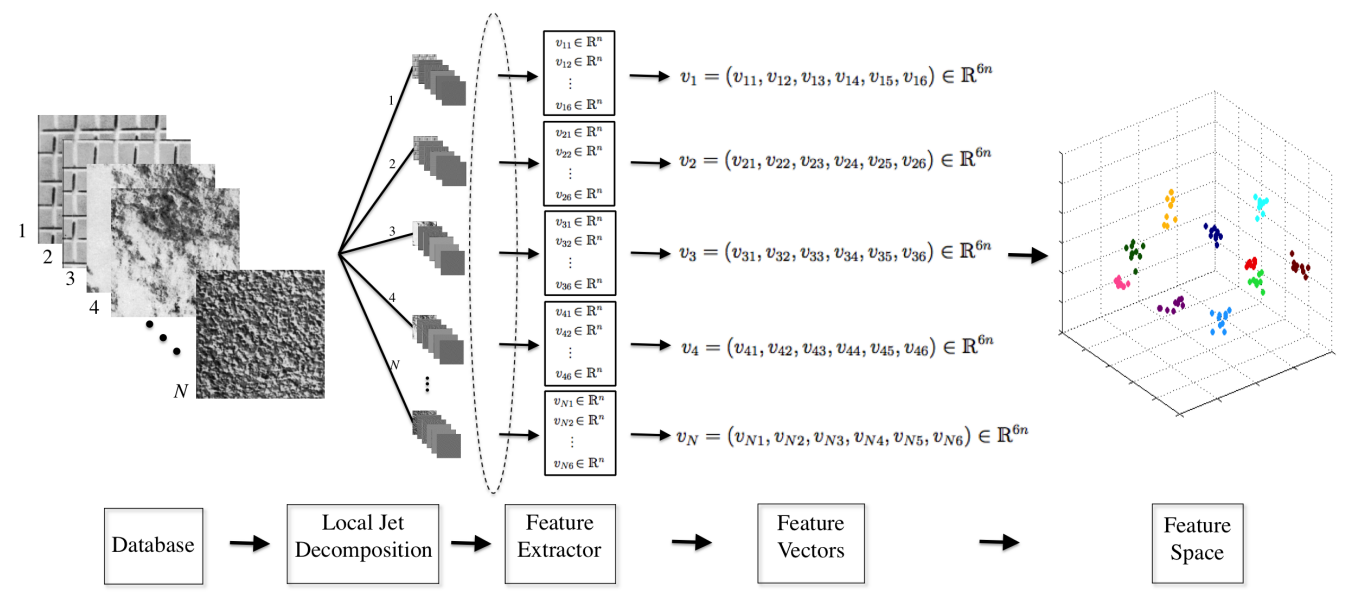

Figure 3: Proposed method scheme. The local jet decomposition is applied to each image of the database resulting in six local jet components normalized in $[0,255]$. Fourier, Gabor, Local Binary Pattern and Local Binary Pattern Variance were used to extract $n$ features from each component generating a feature vector $v_{i} \in \mathbb{R}^{6 n}$. The technique for dimension reduction PCA is applied to the set of vectors of dimension $6 n$ for each texture descriptor to obtain the coordinates with greatest variability. The feature space is composed by these transformed feature vectors.

of circular rings, and $E b$ is the energy from circular regions, defined as

$$
\begin{gathered}
E a_{i j}=\int_{\lambda_{i}}^{\lambda_{i+1}} \int_{\theta_{j}}^{\theta_{j+1}}|F(\lambda, \theta)|^{2} d \theta d \lambda, \\
E b_{i}=\int_{0}^{\lambda_{i}} \int_{0}^{2 \pi}|F(\lambda, \theta)|^{2} d \theta d \lambda,
\end{gathered}
$$

where $(\lambda, \theta)$ are the polar coordinates of points in the frequency domain, and $\left\{\lambda_{i}\right\}_{i=1}^{M}$ and $\left\{\theta_{j}\right\}_{j=1}^{N}$ are the sets of parameters for the method with $M$ radius and $N$ orientations. The texture image is wholly represented by the Fourier descriptor vector

$$
F D=\left[E a_{11}, \ldots, E a_{M N}, E b_{1}, \ldots, E b_{M}\right]
$$

\subsection{Gabor filters descriptors}

Gabor filters $[12,21,22]$ may identify edges in images according to a set of orientations and scales, as well as local image microstructures. This last property is applied to characterize texture images. 
Formally, a 2D Gabor filter is a bidimensional Gaussian function modulated by sine and cosine function [22,21]. The Gabor functions $g(x, y)$ and its Fourier transform $G(u, v)$ can be defined as

$$
\begin{gathered}
g(x, y)=\frac{1}{2 \pi \sigma_{x} \sigma_{y}} \exp \left[-\frac{1}{2}\left(\frac{x^{2}}{\sigma_{x}^{2}}+\frac{y^{2}}{\sigma_{y}^{2}}\right)+2 \pi j W x\right], \\
G(u, v)=\exp \left[-\frac{1}{2}\left(\frac{(u-W)^{2}}{\sigma_{w}^{2}}+\frac{v^{2}}{\sigma_{v}^{2}}\right)\right],
\end{gathered}
$$

where $\sigma_{u}=\frac{\pi \sigma_{x}}{2}$ and $\sigma_{v}=\frac{\pi \sigma_{y}}{2}$. Based on $g(x, y)$, the filter set can be obtained through dilations and rotations, i.e.,

$$
\begin{gathered}
g_{m n}(x, y)=a^{-m} G\left(x^{\prime}, y^{\prime}\right), \quad \text { with } a>1, m, n \in \mathbb{N}, \\
x^{\prime}=a^{-m}(x \cos \theta+y \sin \theta), \text { and } \quad y^{\prime}=a^{-m}(-x \sin \theta+y \cos \theta),
\end{gathered}
$$

where $\theta=\frac{n \pi}{N}$ and $N$ is the number of orientations.

The feature extraction procedure is based on the convolution of the texture image by a bank of Gabor filters with $M$ scales and $N$ orientations previously defined [12]. In the frequency domain, the spectrum energy $E$ is a general descriptor. Then, the feature vector is composed by the spectrum energy of each convolution. The texture image is wholly represented by the Gabor filter descriptors vector

$$
G F D=\left[E_{11}, E_{12}, \ldots, E_{21}, \ldots, E_{M N}\right] .
$$

\subsection{Local Binary Pattern}

Proposed by Ojala et al. [23, 15], Local Binary Pattern $(L B P)$ is a simple yet efficient operator to analyze texture images. This operator characterizes the spatial structure based on local image binary patterns. For each pixel $(i, j)$ in image, the $L B P$ code is computed by

$$
L B P_{P, R}(i, j)=\sum_{p=0}^{P-1} s\left(g_{p}-g_{c}\right) 2^{p}, \text { where } s(x)= \begin{cases}1, & \text { if } x \geq 0 \\ 0, & \text { if } x<0,\end{cases}
$$

$g_{c}$ is the gray level of central pixel $(i, j), g_{p}$ is the gray level of the $p$ neighbors $(p=0, \ldots, P-1)$ and $R$ is the radius of the neighborhood. The matrix with 
the $L B P$ code of each pixel is the $L B P$ map of the image and the histogram of the LBP map globally represents the texture.

In [15], Ojala et al. define a uniformity measure $U$

$U\left(L B P_{P, R}(i, j)\right)=\left|s\left(g_{p-1}-g_{c}\right)-s\left(g_{0}-g_{c}\right)\right|+\sum_{p=1}^{P-1}\left|s\left(g_{p}-g_{c}\right)-s\left(g_{p-1}-g_{c}\right)\right|$

and a contrast and rotation invariant texture descriptor using

$$
\operatorname{LBP}_{P, R}^{r i u 2}(i, j)= \begin{cases}\sum_{p=0}^{P-1} s\left(g_{p}-g_{c}\right), & \text { if } U\left(L B P_{P, R}(i, j)\right) \leq 2 \\ P+1, & \text { otherwise }\end{cases}
$$

In our study, we consider the histogram of original $L B P$ code to describe the texture image, and the rotation invariance is left only to the descriptor $L B P V$ as described below.

\subsection{Local Binary Pattern Variance}

Ojala et al. [15] suggest the use of variance measure $V A R_{P, R}$ to incorporate the contrast of local image texture information to $L B P_{P, R}^{\text {riu2 }}$ operator. Hence,

$$
V A R_{P, R}=\frac{1}{P} \sum_{p=0}^{P-1}\left(g_{p}-\mu\right)^{2}, \quad \text { where } \mu=\frac{1}{P} \sum_{p=0}^{P-1} g_{p}
$$

is combined with $L B P_{P, R}^{r i u 2}$ to obtain a powerful rotation invariant operator for local image texture analysis.

However, Guo et al. [24] show limitations of using $V A R_{P, R}$ by having continuous values that need to be quantized. This situation leads to the issue of choosing the number of bins of the histogram. On the one hand, if too few bins are used, they could not be enough to represent the image. On the other hand, if too many bins are used, it could generate a sparse or unstable histogram. Alternatively, Guo et al. propose the use of $V A R_{P, R}$ as an adaptive weight in histogram calculation of $L B P$ code. Let the texture image be a $M \times N$ matrix with maximal $L B P$ pattern value equal $K$, the whole texture image is represented by the Local Binary Pattern Variance $(L B P V)$ histogram 


$$
\begin{array}{r}
L B P V_{P, R}(k)=\sum_{i=1}^{M} \sum_{j=1}^{N} w\left(L B P_{P, R}^{r i u 2}(i, j), k\right), \quad k \in[0, K], \\
w\left(L B P_{P, R}^{r i u 2}(i, j), k\right)= \begin{cases}V A R_{P, R}(i, j), & \text { if } L B P_{P, R}^{r i u 2}(i, j)=k, \\
0, & \text { otherwise. }\end{cases}
\end{array}
$$

\section{Experiments}

In this section, we show the various experiments that were carried out to demonstrate the better performance of extracting features from local jet space for texture classification instead of extracting features directly from the original images. In our experiments, we have used four databases: Brodatz, Vistex, Usptex and Outex, described in Table 1 with number of classes, number of images in all databases, number of images per class and size of each image. The entirety of each database was used in the experiments.

Table 1: Databases.

\begin{tabular}{lcccc}
\hline Database & \# Classes & \# Images & \# Images per class & \# Image size \\
\hline Brodatz [25] & 111 & 1110 & 10 & $200 \times 200$ pixels \\
Vistex [14] & 54 & 864 & 16 & $128 \times 128$ pixels \\
Usptex [13] & 191 & 2292 & 12 & $128 \times 128$ pixels \\
Outex [26] & 68 & 1360 & 20 & $128 \times 128$ pixels \\
\hline
\end{tabular}

To demonstrate the efficiency of our proposed approach, we have extracted features from images without preprocessing and we have compared with the results of extracting features over the local jet space. Fourier descriptors, Gabor filters, Local Binary Pattern and Local Binary Pattern Variance, as explained before in Section 3, were used in the experiments. Moreover, another set of experiments were conducted to verify the robustness of the proposed approach in front of noise images. To achieve this goal, the Gaussian noise, which appears during the process of image acquisition caused due poor illumination, electronic circuit noise, among others. Three different versions of each database have been generated using intensities of the Gaussian noise with zero mean and variance $\sigma$ equals to $0.01,0.05$, e 0.1 . Figure 4 shows samples of the modified databases with noise. 

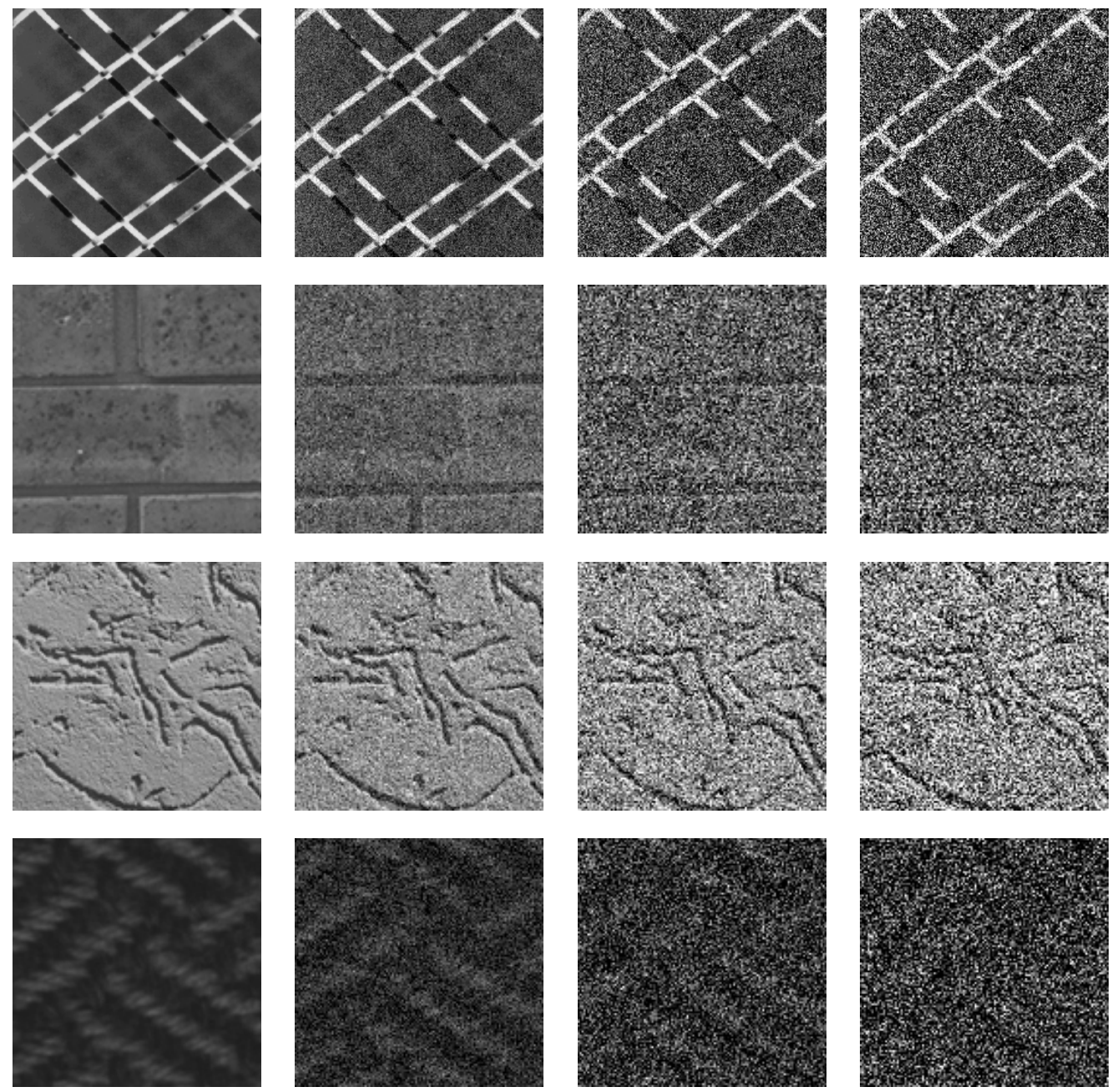

Figure 4: Image samples from the modified databases with Gaussian noise. Each row shows one image from the Brodatz, Vistex, Usptex and Outex databases, from top to bottom. First column represents the original image and the remainder columns represent the images with Gaussian noise zero mean and variance $\sigma=0.01,0.05$ e 0.1 , from left to the right.

As the feature space is a subset of $\mathbb{R}^{6 n}$, a dimensionality reduction method called Principal Components Analysis (PCA) was used. PCA converts a set of features extracted from image in orthogonal components to each others, so there is no redundant information. The transformed vector is as large as the 
original vector, however, that vector is a set of linearly uncorrelated values, sorted according to the variance of the original data [16]. In all cases, the classification was performed using Linear Discriminant Analysis [27] over the feature space transformed by PCA. The validation scheme of classification was stratified 10-fold cross-validation [28]. The experiments of comparison (i.e. the traditional methodology of texture extraction) were also conducted with PCA transformation over the feature space and classification using LDA, under stratified 10-fold cross-validation scheme.

\section{Results and Discussions}

For pattern recognition purpose, one of the ways of evaluating a feature extractor is to look at the dimension of descriptors. The lower the dimension of features, the more efficient the feature extractor. For this reason we analyse the success rate according to the dimension of features for each feature extraction method used here compared to the proposed local jet approach combining feature extraction method. Furthermore, these plots are shown for each database used in the experiments. Thus, it will be shown 16 graphics, being four databases (Brodatz, Vistex, Usptex and Outex) and four feature extractors (Fourier descriptors, Gabor filters, Local Binary Pattern and Local Binary Pattern Variance), as can be seen at Figures 5-8. It can also be observed in these figures that the behavior of the curves is similar with respect to the increase of the dimension of descriptors ( $x$-axis). Moreover, the proposed approach has better success rates compared to traditional methodology with low dimension of descriptors.

The results can be summarized and analyzed in two modes: with a fixed dimension of descriptors or comparing the best results, regardless of the dimension of descriptors. Considering the same dimension, the proposed approach has success rate higher than the approaches used in traditional way, as detailed in Figures 5-8 and Table 2. Here, the best improvement is the application of local jet associated with the Fourier descriptor in Usptex database. If we consider only the best success rate, ignoring the dimension of descriptors, the success rate rises considerably according to Table 3. In this regard, the proposed approach was responsible for an average increase of 2.6 percent in success rate for all traditional methods.

It is noticeable that in almost all experiments using local jet space resulted in a higher success rate. Two main observations should be done, the first is related to results by combining the local jet space with LBP method, 


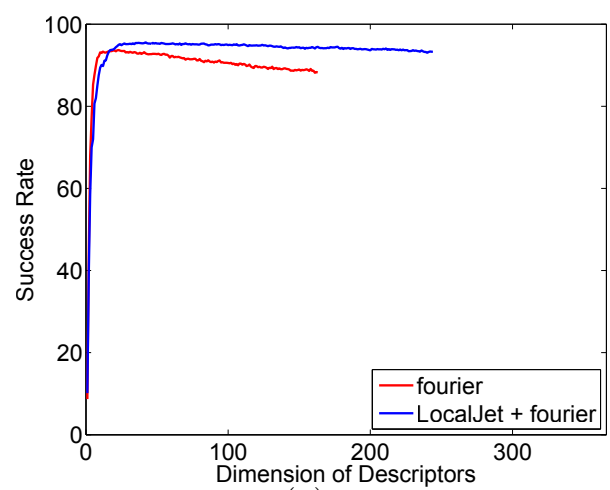

(a)

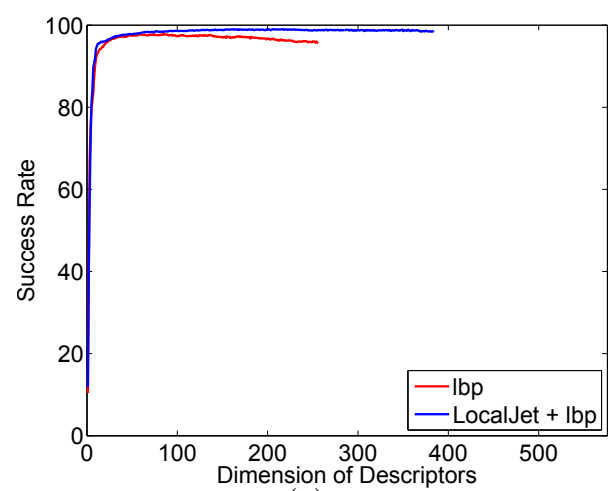

(c)

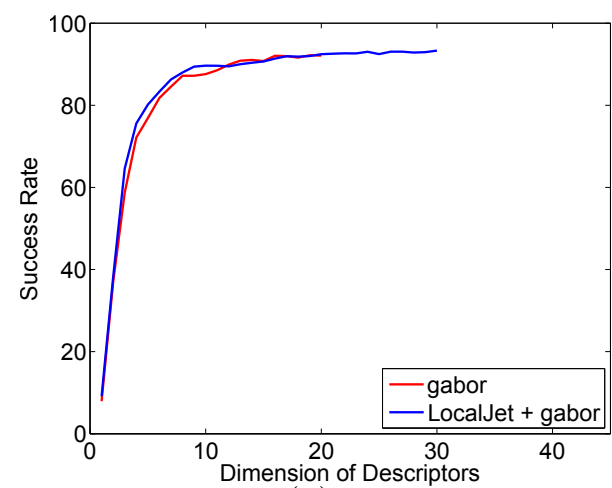

(b)

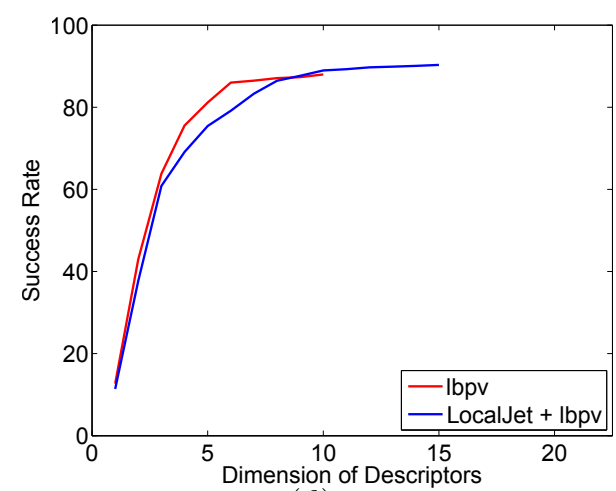

(d)

Figure 5: Dimension of descriptors $\times$ Success rate comparing the traditional features extraction and feature extraction on local jet space for Brodatz database.

Table 2: Success rate of all experiments using the same dimension of descriptors. \# represents the dimension of descriptors and * means 163 descriptors for Brodatz database.

\begin{tabular}{|c|c|c|c|c|c|}
\hline \multirow{3}{*}{ Method/Database } & \multirow{3}{*}{ \# } & \multicolumn{4}{|c|}{ Success rate $(\%) /( \pm$ std $)$} \\
\hline & & Brodatz & Vistex & Usptex & Outex \\
\hline & & $\% \quad( \pm \mathrm{std})$ & $( \pm$ std $)$ & $( \pm$ std $)$ & $( \pm$ std $)$ \\
\hline Fourier & $127^{*}$ & $88.22( \pm 0.52)$ & $90.19( \pm 0.66)$ & $74.67( \pm 0.24)$ & $82.05( \pm 0.36)$ \\
\hline Local jet + Fourier & $127^{*}$ & $94.49( \pm 0.37)$ & $97.09( \pm 0.28)$ & $87.28( \pm 0.28)$ & $86.04( \pm 0.30)$ \\
\hline Gabor & 20 & $92.09( \pm 0.33)$ & $89.38( \pm 0.47)$ & $81.80( \pm 0.36)$ & $82.82( \pm 0.41)$ \\
\hline Local jet + Gabor & 20 & $92.44( \pm 0.36)$ & $93.46( \pm 0.37)$ & $85.11( \pm 0.19)$ & $83.12( \pm 0.37)$ \\
\hline $\mathrm{LBP}$ & 256 & $95.62( \pm 0.32)$ & $97.34( \pm 0.27)$ & $84.43( \pm 0.39)$ & $79.83( \pm 0.59)$ \\
\hline Local jet + LBP & 256 & 98.81( \pm 0.20$)$ & 99.17( \pm 0.18$)$ & $\mathbf{9 1 . 5 2}( \pm 0.40)$ & $\mathbf{8 6 . 7 3}( \pm 0.56)$ \\
\hline LBPV & 10 & $87.99( \pm 0.34)$ & $81.35( \pm 0.46)$ & $73.65( \pm 0.34)$ & $64.20( \pm 0.43)$ \\
\hline Local jet + LBPV & 10 & $88.96( \pm 0.43)$ & $87.12( \pm 0.39)$ & $66.53( \pm 0.28)$ & $57.30( \pm 0.60)$ \\
\hline
\end{tabular}




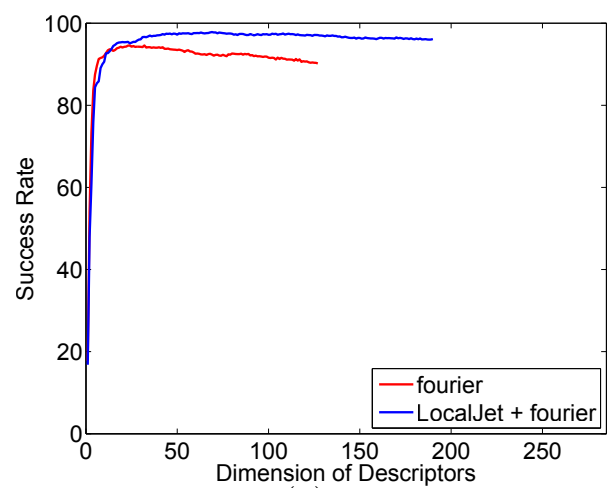

(a)

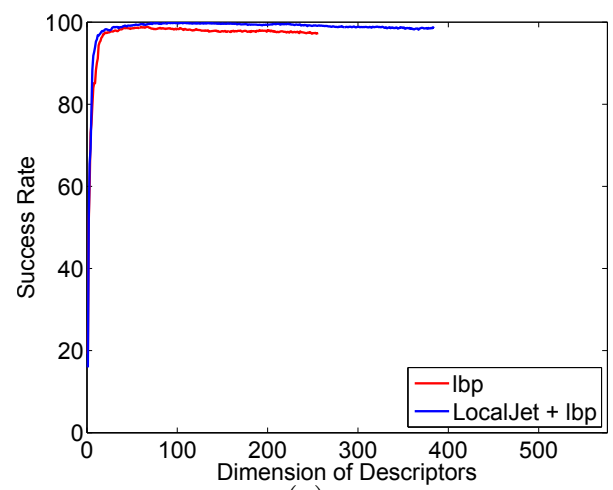

(c)

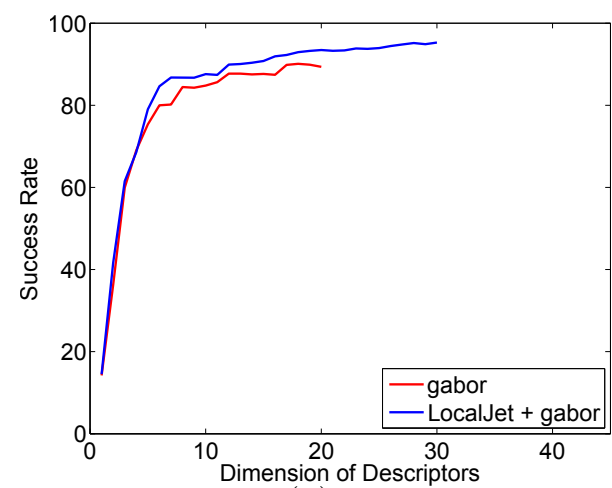

(b)

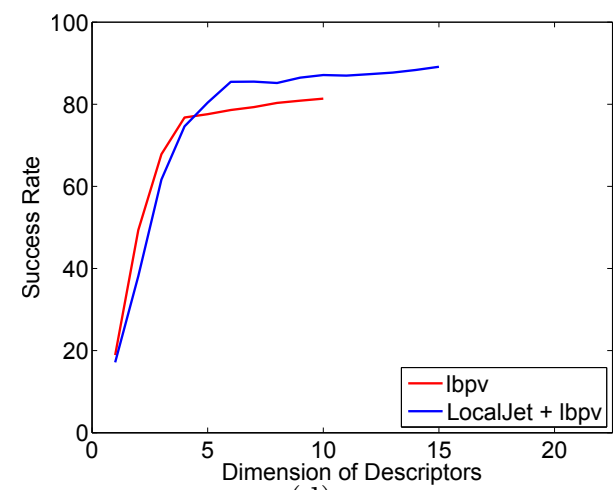

(d)

Figure 6: Dimension of descriptors $\times$ Success rate comparing the traditional features extraction and feature extraction on local jet space for Vistex database.

Table 3: The best success rate of each experiment. \# represents the dimension of descriptors.

\begin{tabular}{|c|c|c|c|c|c|c|c|c|}
\hline \multirow{3}{*}{ Method/Database } & \multicolumn{8}{|c|}{ Dimension of descriptors $(\#) /$ Success rate $(\%) /( \pm$ std $)$} \\
\hline & \multicolumn{2}{|c|}{ Brodatz } & \multicolumn{2}{|c|}{ Vistex } & \multicolumn{2}{|c|}{ Usptex } & \multicolumn{2}{|c|}{ Outex } \\
\hline & \# & $\%( \pm$ std $)$ & $\#$ & $\%( \pm$ std $)$ & $\#$ & $\%( \pm$ std $)$ & $\#$ & $\%( \pm$ std $)$ \\
\hline Fourier & 23 & $93.69( \pm 0.17)$ & 24 & $94.56( \pm 0.28)$ & 18 & $85.01( \pm 0.31)$ & 25 & $85.32( \pm 0.42)$ \\
\hline Local jet + Fourier & 42 & $95.56( \pm 0.23)$ & 69 & $97.84( \pm 0.14)$ & 50 & $89.63( \pm 0.24)$ & 63 & $87.16( \pm 0.43)$ \\
\hline Gabor & 19 & $92.18( \pm 0.35)$ & 18 & $90.09( \pm 0.25)$ & 20 & $81.80( \pm 0.36)$ & 19 & $82.96( \pm 0.62)$ \\
\hline Local jet + Gabor & 30 & $93.31( \pm 0.28)$ & 30 & $95.28( \pm 0.36)$ & 30 & $87.81( \pm 0.23)$ & 29 & $85.46( \pm 0.31)$ \\
\hline LBP & 84 & $97.86( \pm 0.16)$ & 60 & $98.87( \pm 0.17)$ & 80 & $90.93( \pm 0.20)$ & 73 & $85.51( \pm 0.41)$ \\
\hline Local jet + LBP & 216 & $99.02( \pm 0.19)$ & 91 & $99.86( \pm 0.16)$ & 104 & $\mathbf{9 4 . 2 9}( \pm 0.23)$ & 56 & $\mathbf{8 8 . 9 8}( \pm 0.22)$ \\
\hline LBPV & 10 & $87.99( \pm 0.34)$ & 10 & $81.35( \pm 0.46)$ & 10 & $73.65( \pm 0.34)$ & 10 & $64.20( \pm 0.43)$ \\
\hline Local jet + LBPV & 15 & $90.32( \pm 0.35)$ & 15 & $89.12( \pm 0.31)$ & 15 & $74.65( \pm 0.30)$ & 15 & $63.06( \pm 0.69)$ \\
\hline
\end{tabular}



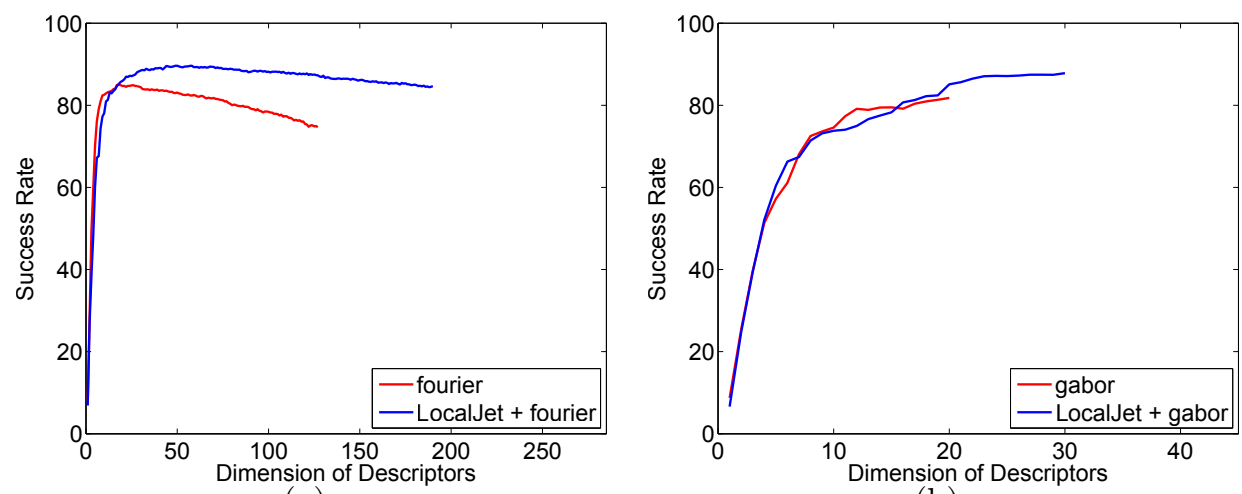

(a)

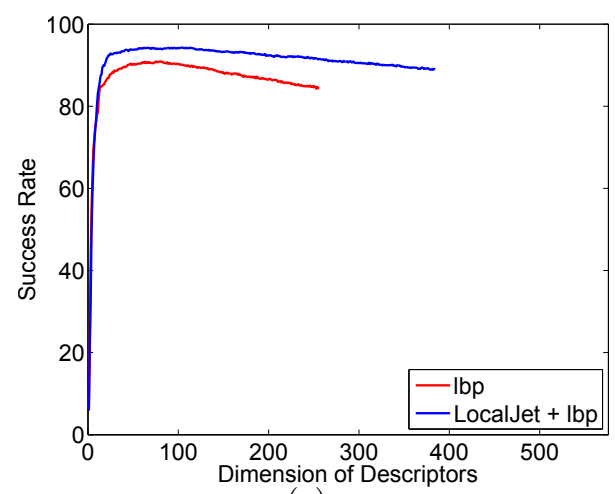

(c)

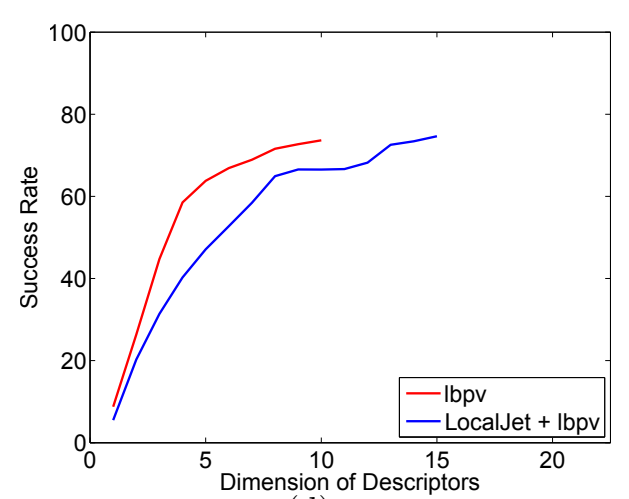

(d)

Figure 7: Dimension of descriptors $\times$ Success rate comparing the traditional features extraction and feature extraction on local jet space for Usptex database.

because this combination produces the best success rate for all cases. The second observation relates to the results obtained in the experiments with Usptex and Outex, that are very difficult databases, where the best result without local jet decomposition is $84.43 \%$ for Usptex and $82.82 \%$ for Outex and using our proposal it was obtained $91.52 \%$ and $86.73 \%$, respectively, according to Table 2 .

Another points to be highlighted concern the results of LBPV, Gabor and Fourier. According to results obtained when using LBPV, which does not show an effective improvement by using the local jet space, coming to have lower success rate in the case of Usptex database for low dimension of descriptors. This is due to LBPV exploits complementary information for 

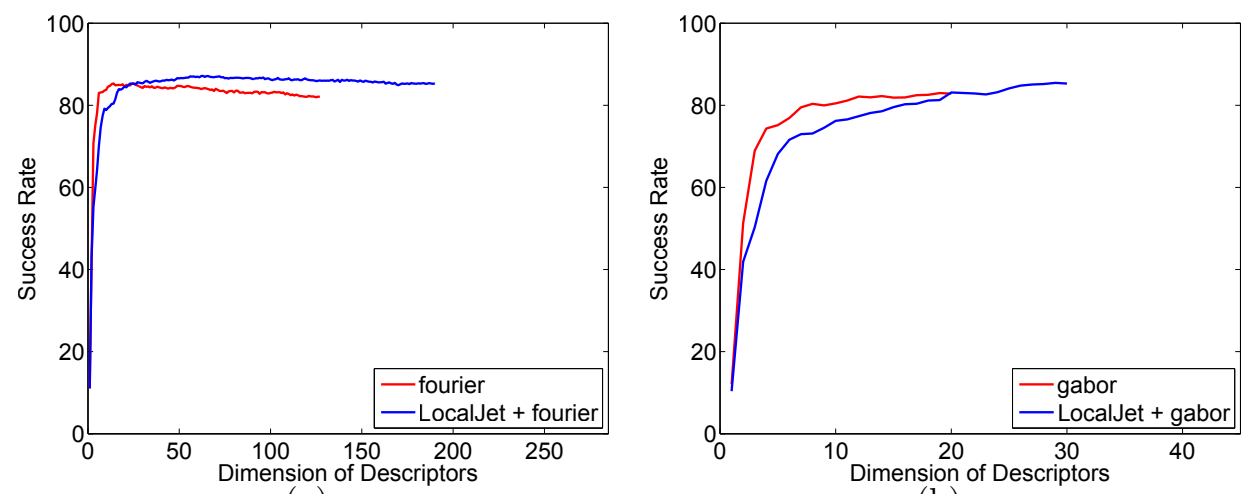

(a)

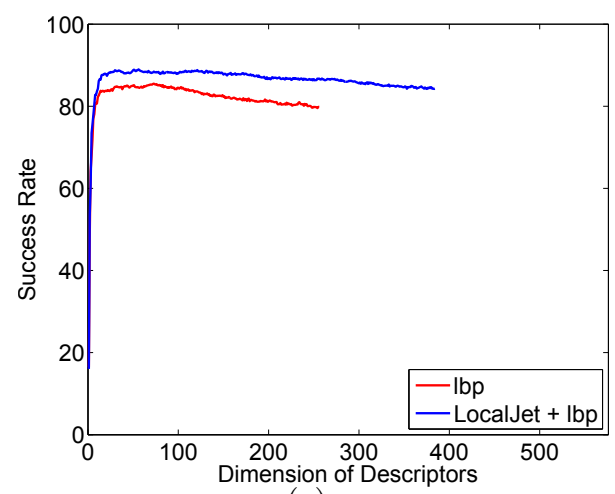

(c)

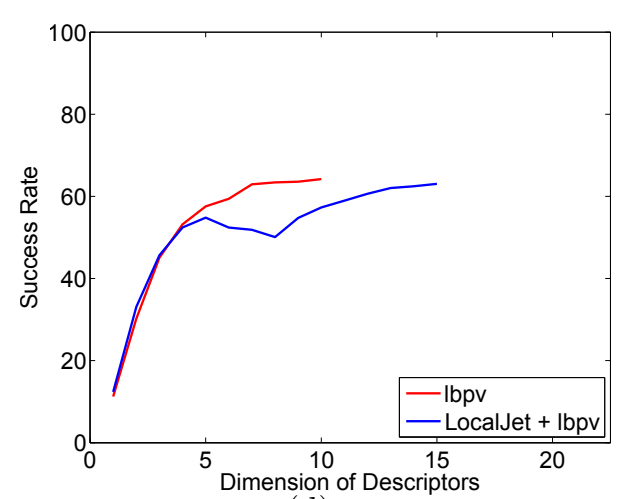

(d)

Figure 8: Dimension of descriptors $\times$ Success rate comparing the traditional features extraction and feature extraction on local jet space for Outex database.

local variance of spatial patterns. However, local jet breaches the geometric patterns in each component reducing the local variance of these patterns. In general, this behavior benefits feature descriptors which disjointly measure the texture patterns. On the other hand, when the texture descriptor analyzes the variation among the patterns this property is less relevant in the local jet space.

Despite the similarity in the geometric interpretation of Gabor filters and local jet, the results of the combination Local jet + Gabor show a considerable increase in the success rate for all tests, as shown at Table 3 . This is due to the fact that when applying the local jet over the image, important directional characteristics are isolated on each component and Gabor descriptors extract 
this information more accurately. In the same direction, Fourier descriptors also make use of the isolated directional characteristics by collecting relevant information in the same frequency points in the Fourier spectrum.

In the experiments related to noise invariance, again, the methodology using local jet space resulted in a higher success rate in most of the cases, as shown at Table 4 . The results presented in Table 4 consider the number of descriptors that produces the best success rate for each experiment. According to the increasing of the Gaussian noise variance, the traditional methods tend to decrease the success rate, mainly when using LBP and LBPV descriptors. On the other hand, at the same time when using the local jet space and the same feature descriptors, the decay of success rate is smoother than in the conventional approach, proving the robustness of local jet space dealing with noisy images.

\section{Conclusions}

In conclusion, we investigated the potential of local jet space to improve the texture characterization by the highlight of the geometric patterns obtained through partial derivatives. In order to carry out this task, we applied classical texture descriptors to the six local jet components, resulting in a feature space of $\mathbb{R}^{6 n}$. To validate the proposal, the experiments were performed in Brodatz, Vistex, Usptex and Outex texture databases using Fourier, Gabor, LBP and LBPV as texture descriptors. In addition, PCA was used for dimensionality reduction, and to compare the new approach with the texture descriptors without using local jets considering feature vectors with the same dimension.

The results from the comparison between texture features extracted from the local jet space and directly from the image indicate the importance of the former, resulting in higher success rates for texture classification. For the particular experiment with Fourier descriptors and Usptex database, we have found the greatest increase of success rate, from $74.67 \%$ to $87.28 \%$, by using of local jet decomposition. More generally, the proposed approach was responsible for an average increase of $2.6 \%$ in success rate for all traditional methods when considering only the best success rate of each experiment. The combination of the local jet space and texture feature extractors, leads us to the results that are promising for the field of pattern recognition and image analysis. 
Table 4: Success rates of texture classification for three databases modified from the original ones obtained through addition of Gaussian noise with zero mean and variance $\sigma$ $=0.01,0.05$ e 0.1 .

\begin{tabular}{|c|c|c|c|c|c|c|c|}
\hline \multirow{3}{*}{ Database } & \multirow{3}{*}{ Method } & \multicolumn{6}{|c|}{ Dimension of descriptors $(\#) /$ Success rate $(\%) /( \pm$ std $)$} \\
\hline & & \multicolumn{2}{|r|}{$l=0.01$} & \multicolumn{2}{|c|}{$l=0.05$} & \multicolumn{2}{|c|}{$l=0.1$} \\
\hline & & $\#$ & $\%( \pm \mathrm{std})$ & \# & $\%( \pm$ std $)$ & $\#$ & $\%( \pm$ std $)$ \\
\hline \multirow{8}{*}{ Brodatz } & Fourier & 23 & $93.25( \pm 0.26)$ & 23 & $91.73( \pm 0.22)$ & 38 & $90.58( \pm 0.33)$ \\
\hline & Local jet + Fourier & 48 & $94.35( \pm 0.25)$ & 24 & $92.03( \pm 0.22)$ & 45 & $91.25( \pm 0.56)$ \\
\hline & Gabor & 19 & $91.20( \pm 0.32)$ & 19 & $90.85( \pm 0.34)$ & 19 & $88.99( \pm 0.47)$ \\
\hline & Local jet + Gabor & 29 & $91.29( \pm 0.49)$ & 29 & $88.59( \pm 0.46)$ & 29 & $87.67( \pm 0.53)$ \\
\hline & LBP & 35 & $92.09( \pm 0.43)$ & 19 & $84.69( \pm 0.45)$ & 11 & $78.06( \pm 0.41)$ \\
\hline & Local jet + LBP & 69 & $95.52( \pm 0.11)$ & 24 & $89.47( \pm 0.20)$ & 22 & $84.92( \pm 0.35)$ \\
\hline & LBPV & 10 & $85.20( \pm 0.37)$ & 10 & $72.57( \pm 0.55)$ & 9 & $62.44( \pm 0.83)$ \\
\hline & Local jet + LBPV & 14 & $84.98( \pm 0.29)$ & 15 & $82.50( \pm 0.38)$ & 14 & $80.06( \pm 0.66)$ \\
\hline \multirow{8}{*}{ Vistex } & Fourier & 24 & $94.03( \pm 0.33)$ & 32 & $89.25( \pm 0.45)$ & 24 & $85.68( \pm 0.56)$ \\
\hline & Local jet + Fourier & 45 & $95.30( \pm 0.27)$ & 47 & $89.10( \pm 0.56)$ & 36 & $83.40( \pm 0.67)$ \\
\hline & Gabor & 18 & $85.64( \pm 0.50)$ & 17 & $80.72( \pm 0.29)$ & 18 & $76.37( \pm 0.49)$ \\
\hline & Local jet + Gabor & 29 & $91.67( \pm 0.66)$ & 30 & $81.16( \pm 0.51)$ & 30 & $74.76( \pm 0.77)$ \\
\hline & LBP & 16 & $84.51( \pm 0.30)$ & 12 & $68.69( \pm 0.38)$ & 22 & $60.16( \pm 0.36)$ \\
\hline & Local jet + LBP & 39 & $91.32( \pm 0.41)$ & 18 & $79.65( \pm 0.34)$ & 7 & $68.55( \pm 0.48)$ \\
\hline & LBPV & 7 & $70.57( \pm 0.44)$ & 9 & $60.24( \pm 0.68)$ & 8 & $53.61( \pm 0.71)$ \\
\hline & Local jet + LBPV & 15 & $82.03( \pm 0.43)$ & 14 & $72.20( \pm 0.68)$ & 14 & $61.93( \pm 0.55)$ \\
\hline \multirow{8}{*}{ Usptex } & Fourier & 25 & $73.99( \pm 0.42)$ & 25 & $56.22( \pm 0.21)$ & 16 & $47.19( \pm 0.33)$ \\
\hline & Local jet + Fourier & 45 & $73.43( \pm 0.40)$ & 50 & $50.00( \pm 0.58)$ & 28 & $41.68( \pm 0.53)$ \\
\hline & Gabor & 17 & $70.66( \pm 0.54)$ & 14 & $53.17( \pm 0.48)$ & 19 & $40.82( \pm 0.25)$ \\
\hline & Local jet + Gabor & 28 & $71.03( \pm 0.41)$ & 30 & $49.40( \pm 0.48)$ & 30 & $38.43( \pm 0.28)$ \\
\hline & LBP & 12 & $44.11( \pm 0.32)$ & 10 & $21.99( \pm 0.31)$ & 22 & $17.12( \pm 0.43)$ \\
\hline & Local jet + LBP & 19 & $69.18( \pm 0.33)$ & 18 & $35.54( \pm 0.48)$ & 8 & $22.86( \pm 0.44)$ \\
\hline & LBPV & 6 & $32.67( \pm 0.45)$ & 8 & $20.03( \pm 0.26)$ & 6 & $17.03( \pm 0.34)$ \\
\hline & Local jet + LBPV & 15 & $49.83( \pm 0.43)$ & 12 & $27.07( \pm 0.37)$ & 14 & $19.11( \pm 0.31)$ \\
\hline \multirow{8}{*}{ Outex } & Fourier & 13 & $71.52( \pm 0.49)$ & 17 & $56.15( \pm 0.52)$ & 29 & $46.37( \pm 0.52)$ \\
\hline & Local jet + Fourier & 52 & $69.29( \pm 0.41)$ & 50 & $51.25( \pm 0.80)$ & 60 & $42.77( \pm 0.72)$ \\
\hline & Gabor & 18 & $69.85( \pm 0.92)$ & 18 & $49.42( \pm 0.45)$ & 17 & $40.22( \pm 0.71)$ \\
\hline & Local jet + Gabor & 30 & $68.06( \pm 0.44)$ & 28 & $46.41( \pm 0.43)$ & 30 & $37.65( \pm 0.51)$ \\
\hline & $\mathrm{LBP}$ & 34 & $28.32( \pm 0.93)$ & 6 & $26.41( \pm 0.81)$ & 3 & $19.99( \pm 0.53)$ \\
\hline & Local jet + LBP & 18 & $44.01( \pm 0.53)$ & 8 & $29.65( \pm 0.49)$ & 12 & $21.76( \pm 0.58)$ \\
\hline & LBPV & 7 & $25.35( \pm 0.79)$ & 3 & $26.10( \pm 0.41)$ & 3 & $19.93( \pm 0.54)$ \\
\hline & Local jet + LBPV & 15 & $36.74( \pm 0.63)$ & 15 & $26.20( \pm 0.35)$ & 12 & $22.16( \pm 0.75)$ \\
\hline
\end{tabular}




\section{References}

[1] L. Florack, B. Ter Haar Romeny, M. Viergever, J. Koenderink, The Gaussian scale-space paradigm and the multiscale local jet, International Journal of Computer Vision 18 (1996) 61-75.

[2] A. Manzanera, Local jet based similarity for NL-means filtering, in: International Conference on Pattern Recognition (ICPR'10), volume 0, IEEE Computer Society, Istambul, Turkey, 2010, pp. 2668-2671.

[3] A. Manzanera, Local jet feature space framework for image processing and representation, in: Signal-Image Technology and Internet-Based Systems (SITIS), 2011 Seventh International Conference on, pp. 261268.

[4] J. Koenderink, A. van Doorn, Representation of local geometry in the visual system, Biological Cybernetics 55 (1987) 367-375.

[5] R. Pydipati, T. Burks, W. Lee, Identification of citrus disease using color texture features and discriminant analysis, Computers and Electronics in Agriculture 52 (2006) 49 - 59.

[6] L. Journaux, J.-C. Simon, M. Destain, F. Cointault, J. Miteran, A. Piron, Plant leaf roughness analysis by texture classification with generalized Fourier descriptors in a dimensionality reduction context, Precision Agriculture 12 (2011) 345-360.

[7] D. R. Rossatto, D. Casanova, R. M. Kolb, O. M. Bruno, Fractal analysis of leaf-texture properties as a tool for taxonomic and identification purposes: a case study with species from Neotropical Melastomataceae (Miconieae tribe), Plant Systematics and Evolution 291 (2011) 103-116.

[8] J. B. Florindo, N. R. da Silva, L. M. Romualdo, F. de Fátima da Silva, P. H. de Cerqueira Luz, V. R. Herling, O. M. Bruno, Brachiaria species identification using imaging techniques based on fractal descriptors, Computers and Electronics in Agriculture 103 (2014) 48 - 54.

[9] G. Castellano, L. Bonilha, L. Li, F. Cendes, Texture analysis of medical images, Clinical Radiology 59 (2004) 1061 - 1069. 
[10] B. Zhou, J. Xuan, H. Zhao, G. Chepko, M. Freedman, K. Y. Zou, Polarization imaging for breast cancer diagnosis using texture analysis and SVM, in: Life Science Systems and Applications Workshop, 2007. LISA 2007. IEEE/NIH, pp. 217-220.

[11] H. Yu, C. Caldwell, K. Mah, D. Mozeg, Coregistered FDG PET/CTbased textural characterization of head and neck cancer for radiation treatment planning, IEEE TRANSACTIONS ON MEDICAL IMAGING 28 (2009).

[12] B. Manjunath, W. Ma, Texture features for browsing and retrieval of image data, Pattern Analysis and Machine Intelligence, IEEE Transactions on 18 (1996) 837-842.

[13] A. R. Backes, D. Casanova, O. M. Bruno, Color texture analysis based on fractal descriptors, Pattern Recognition 45 (2012) 1984-1992.

[14] Vision texture database, Maintained by the Vision and Modeling group at the MIT Media Lab. http://whitechapel.media.mit.edu/vismod/, 2009.

[15] T. Ojala, M. Pietikäinen, T. Mäenpää, Multiresolution gray-scale and rotation invariant texture classification with local binary patterns, IEEE Transactions Pattern Analysis and Machine Intelligence 24 (2002) 971987.

[16] I. T. Jolliffe, Principal component analysis, Springer, New York, 2002.

[17] C. T. Zahn, R. Z. Roskies, Fourier descriptors for plane closed curves, Transactions on Computers, IEEE c-21 (1972) 269-281.

[18] R. Azencott, J.-P. Wang, L. Younes, Texture classification using windowed Fourier filters, Pattern Analysis and Machine Intelligence, IEEE Transactions on 19 (1997) $148-153$.

[19] R. C. Gonzalez, R. E. Woods, Digital Image Processing (3rd Edition), Prentice-Hall, Inc., Upper Saddle River, NJ, USA, 2006.

[20] L. Journaux, M.-F. Destain, J. Mitéran, A. Piron, F. Cointault, Texture classification with generalized fourier descriptors in dimensionality 
reduction context: An overview exploration., in: L. Prevost, S. Marinai, F. Schwenker (Eds.), ANNPR, volume 5064 of Lecture Notes in Computer Science, Springer, 2008, pp. 280-291.

[21] J. Daugman, C. Downing, Gabor wavelets and statistical pattern recognition, in: M. A. Arbib (Ed.), The handbook of brain theory and neural networks, MIT Press, Cambridge, MA, USA, 1998, pp. 414-420.

[22] M. Idrissa, M. Acheroy, Texture classification using Gabor filters, Pattern Recognition Letters 23 (2002) 1095-1102.

[23] T. Ojala, M. Pietikäinen, D. Harwood, A comparative study of texture measures with classification based on featured distributions, Pattern Recognition 29 (1996) 51-59.

[24] Z. Guo, L. Zhang, D. Zhang, Rotation invariant texture classification using LBP variance (LBPV) with global matching, Pattern Recognition 43 (2010) 706-719.

[25] P. Brodatz, Textures; a photographic album for artists and designers, Dover Publications New York, 1966.

[26] T. Ojala, T. Mäenpää, M. Pietikäinen, J. Viertola, J. Kyllönen, S. Huovinen, Outex - new framework for empirical evaluation of texture analysis algorithms, in: Proceedings of the 16 th International Conference on Pattern Recognition (ICPR'02) Volume 1 - Volume 1, ICPR '02, IEEE Computer Society, Washington, DC, USA, 2002, p. 10701.

[27] R. A. Fisher, The use of multiple measurements in taxonomic problems, Annals of Eugenics 7 (1936) 179-188.

[28] T. Hastie, R. Tibshirani, J. Friedman, The Elements of Statistical Learning, Springer Series in Statistics, Springer New York Inc., New York, NY, USA, 2001. 